\title{
New target solution for a muon collider or a muon-decay neutrino beam facility: The granular waterfall target
}

\author{
Han-Jie Cai, ${ }^{1,2}$ Guanghui Yang, ${ }^{1,2}$ Nikos Vassilopoulos, ${ }^{3}$ Sheng Zhang, ${ }^{1}$ Fen Fu, ${ }^{1}$ \\ Ye Yuan, ${ }^{3}$ and Lei Yang, \\ ${ }^{1}$ Institute of Modern Physics, CAS, Lanzhou 730000, China \\ ${ }^{2}$ University of Chinese Academy of Sciences, Beijing 100049, China \\ ${ }^{3}$ Institute of High Energy Physics, CAS, Beijing 100049, China
}

(Received 13 July 2016; published 1 February 2017)

\begin{abstract}
A new target solution, the granular waterfall target, is proposed here for a muon collider or a muon-decay neutrino beam facility, especially for the moment which adopts a 15 MW continuous-wave (cw) superconducting linac. Compared to the mercury jet target, the granular waterfall target works by a much simpler mechanism which can operate with a much more powerful beam, which are indicated by the detailed investigations into the heat depositions and the evaluations of the temperature increases for different target concepts. By varying proton beam kinetic energy and the geometrical parameters of the waterfall target, an overall understanding of the figure of merit concerning muon production for this target concept as the target solutions of the long-baseline neutrino factory and the medium-baseline moment is obtained. With $8 \mathrm{GeV}$ beam energy and the optimal geometrical parameters, the influence on muon yield by adopting different beam-target interaction parameters is explored. Studies and discussions of the design details concerning beam dumping are also presented.
\end{abstract}

DOI: 10.1103/PhysRevAccelBeams.20.023401

\section{INTRODUCTION}

For a muon collider [1] or a muon-decay neutrino beam facility, such as the neutrino factory (NF) $[2,3]$ and the MOMENT [4], the requirement of high intensity muon source requires that the target station be capable of surviving with the bombardment of a proton beam with multi- even tens-of-MW power. Since the conversion rate of the proton beam into the muon source mainly depends on the collection efficiency of the pions produced in the target by the proton-target interaction, the transverse dimension of the target should be small to facilitate the emission of the pions. The high beam power and the small target size lead to an extremely high power density, which poses significant challenges to the target station.

For the muon collider or the neutrino factory which adopts a 4-MW pulsed proton beam [1,3], a free-flowing liquid mercury jet has been proposed to act as the target $[5,6]$. The technical feasibility of the mercury jet target has been demonstrated by the MERIT experiment [7]. For a liquid mercury jet target, more than $10 \%$ of beam power (4 MW) is deposited in the jet $[8,9]$. With a $20 \mathrm{~m} / \mathrm{s}$ jet velocity $[10,11]$ and an 8 -mm jet diameter $[9,12]$, the

\footnotetext{
Corresponding author. lyang@impcas.ac.cn

Published by the American Physical Society under the terms of the Creative Commons Attribution 3.0 License. Further distribution of this work must maintain attribution to the author(s) and the published article's title, journal citation, and DOI.
}

temperature rise will be around $210 \mathrm{~K}$. When the mercury jet enters the target volume at room temperature, it will not be vaporized after the beam-jet interaction. Therefore, it is believed that the mercury jet target can handle the power deposition of a $4 \mathrm{MW}$ proton beam. However, the temperature will not be much lower than the boiling point of mercury $\left(\sim 357^{\circ} \mathrm{C}\right)$. When a much more powerful beam is mandatory, just like what has been proposed (15 MW even more) for the muon-decay medium-baseline neutrino beam facility (MOMENT) project [4,13], the jet velocity should be as high as $75 \mathrm{~m} / \mathrm{s}$. It is unlikely that a liquid metal jet can work stably and properly with such a high velocity, even under the constraint of a magnetic field. On the one hand, the magnetic field as strong as 15 even $20 \mathrm{~T}$ can significantly reduce the amplitudes and velocities of the surface instabilities (Richtmyer-Meshkov instability) of the liquid metal jet $[10,14,15]$. On the other hand, the loss of jet velocity due to the stopping forces and the transformation of the shape of jet caused by the quadrupole effect would grow along with the increasing of the jet velocity and the magnetic field [10,16-18]. Based on the above considerations, a velocity of around $20 \mathrm{~m} / \mathrm{s}$ is thought to be appropriate for a liquid mercury jet target $[9-12,16]$.

In addition to the power limits, the liquid metal jet fails in the continuous-wave $(\mathrm{cw})$ beam design. For a liquid metal jet target, a proton beam will disrupt the jet over most of its effective length and special bunch separation of the pulsed beam is necessary for the liquid metal jet to recovery from the disruption [3,12]. In a word, it is necessary for us to look for a new target solution for the MOMENT which will 


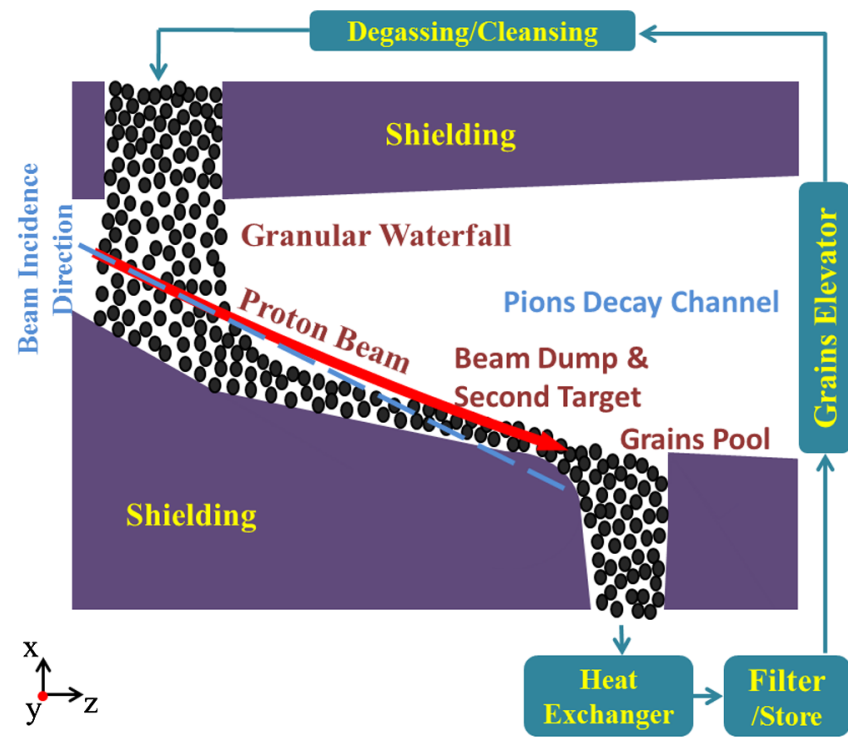

FIG. 1. Schematic outline of a granular waterfall target in the grain loop system with heat exchanger, grain filter, lift, and degassing/cleansing devices.

adopt a cw superconducting linac [13] similar to that for the China-ADS project [19].

We propose here a new target solution, the granular waterfall target, as shown in Fig. 1, for a muon-decay neutrino beam facility. This kind of target concept adopts a grain waterfall to serve as the target body. Due to its high power-processing ability, the granular waterfall target has the potential to operate with the $15 \mathrm{MW} \mathrm{cw}$ proton beam of the MOMENT. In addition, because of its relatively simple working mechanism, this kind of target concept also can be chosen as an alternative to the traditional mercury jet target. In this paper, a series of simulation studies of the performance of the granular tungsten waterfall target concerning power handling ability and muon yield are presented. In addition, the essential design details such as beam-target interaction parameters are discussed.

\section{TARGET CONCEPT}

The schematic of a granular waterfall target is shown in Fig. 1. The solid granular particles flow through the narrow outlet of an upright cubic hopper to form a waterfall of grains. From the hopper outlet, the waterfall is accelerated by gravity to pass through the beam-target interaction region quickly. When the waterfall falls to the inner surface of the shielding, the grains will flow through the incline chute to reach the downstream pool and circulate in a loop system which contains a heat exchanger, a grain filter, a lift device and a degassing/decontamination equipment. In the incline chute, the grain flow can act as a beam dump as well as a second target.

For a muon collider or a muon-decay neutrino beam facility, the damage caused by the failure of the target is

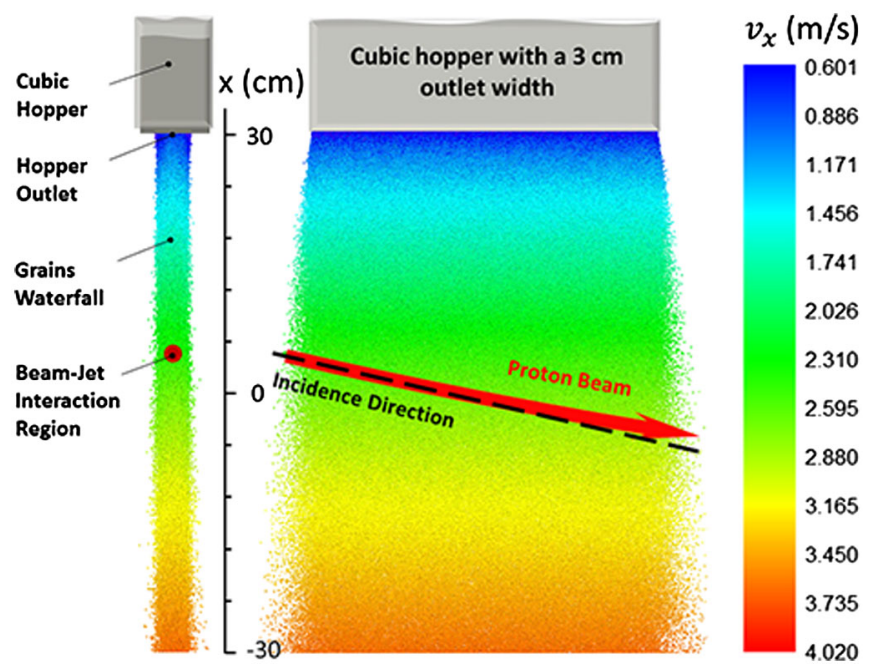

FIG. 2. Schematic representation of beam-target interaction for a granular waterfall target. The velocity variance of the waterfall is shown.

significant $[9,20]$. Therefore, a high reliability is essential for the target system. Compared to the unconstrained liquid metal jet produced by a hydraulically actuated syringe pump [10], a waterfall of granular particles works by a much simpler mechanism with favorable inherent safety due to the fact that the waterfall is formed by the gravitydriven flow of granular particles stacked in the upright hopper, as shown in Fig. 2. Thus, the performance of a gravity-driven waterfall target concerning safety seems to be better than that of a mercury jet target.

Another advantage of the grain waterfall is its high flow rate. Compared to the slender liquid metal jet, the stream cross section of the waterfall is much larger, which is essential to the acquirement of a high flow rate when the velocities for both targets are limited. With a larger flow rate, it can be expected that the granular waterfall be capable of operating with a higher beam power, especially when the high melting temperature of tungsten is taken into consideration. The detailed comparisons between the jet targets and the grain waterfall target are presented in this section.

For the studies of the grain waterfall target, the investigations into the granular flow/jet are necessary. Owing to the R\&D efforts [21-25] on the granular flow spallation target [26] for the China accelerator driven subcritical system (C-ADS) project [19], an overall understanding of this kind of granular flow/jet target have been obtained. In this paper, a GPU accelerated [27,28] discrete element method (DEM) $[29,30]$, which has been widely accepted as an effective method for numerical studies of granular materials in static packings or flows [31-34], was used to perform the numerical simulations of the grain waterfall. In this work, the Hertz-Mindlin model $[35,36]$ was applied to perform the calculations of the contact forces among the 
grains and the Velocity-Verlet scheme [37] was used to carried out the integrations of the motion equations.

Using the GPU-accelerated DEM code, a series of numerical simulations of the granular waterfall are performed. The DEM simulations indicate that the grain waterfall keeps in a quite compact shape within tens of $\mathrm{cm}$ in gravity direction ( $\mathrm{x}$ axis in this paper) when the ratio of the outlet width to the grain diameter is significantly larger than 10. In this work, a ratio of 20 is adopted.

For a liquid mercury jet target, as mentioned above, a jet diameter as small as $8 \mathrm{~mm}$ or so is adopted to achieve a high collection efficiency of charged pions and a jet velocity as high as $20 \mathrm{~m} / \mathrm{s}$ is mandatory to handle the power deposition. For a grain waterfall target, to make sure that there is enough room for the helical motions of pions, the beam incidence height is set to be $30 \mathrm{~cm}$ (can be further optimized for a high muons yield, based on detailed beam-target configurations includes beam incident direction and waterfall parameters) below the hopper outlet, as shown in Fig. 2. With this beam incidence position, the optimal waterfall width (outlet width) for a high muons yield will be $\sim 2 \mathrm{~cm}$ for the $\mathrm{NF}$ and $\sim 3 \mathrm{~cm}$ for the MOMENT. To illustrate the potential of operating with the $15 \mathrm{MW}$ proton beam of the MOMENT for the granular tungsten waterfall target, the $3 \mathrm{~cm}$ width is chosen here for the investigations into flow rate and temperature increase.

For comparison, the temperature increases of the materials in the jets (includes liquid mercury jet and powdery tungsten jet) and the granular tungsten waterfall under the bombardment of a $4 \mathrm{MW}$ proton beam $(8 \mathrm{GeV} \& 0.5 \mathrm{~mA})$ are evaluated based on the simulation results of the energy deposit using the Geant4 toolkit [38] and the flow rate data.

The parameters of the beam profile and the target geometry are listed in Table I. The jets are modeled as what have been designed for the NF $[9,12]$ while the grain waterfall is fed from a $3 \mathrm{~cm}$ wide and $50 \mathrm{~cm}$ long hopper outlet. When a Gaussian beam profile is used, the power dissipation in the target is transversely inhomogeneous. As listed in Table I, the beam profile for the jets is Gaussian with a $1.2-\mathrm{mm}$ root mean square (rms) radius while that for the waterfall is uniform in $\mathrm{x}$ direction and Gaussian in y direction.

TABLE I. Parameters of the beam profile and the target geometry.

\begin{tabular}{lcc}
\hline \hline Parameter & $\mathrm{Hg} / \mathrm{W}$ jet & Grains waterfall \\
\hline Beam profile & Gaussian $\mathrm{r}(1.2 \mathrm{~mm})$ & $\begin{array}{c}\text { Gaussian y }(1.2 \mathrm{~mm}) \\
\text { Uniform x }(1 \mathrm{~cm})\end{array}$ \\
Target dimension & $\begin{array}{c}\text { D8xL200 } \mathrm{mm}(\mathrm{Hg}) \\
\text { Y3xZ500 mm }\end{array}$ & \\
Relative density & $1(\mathrm{Hg})$ & As shown in Fig. 6 \\
factor & $0.5(\mathrm{~W})$ & \\
velocity & $20 \mathrm{~m} / \mathrm{s}$ & As shown in Fig. 2 \\
\hline \hline
\end{tabular}

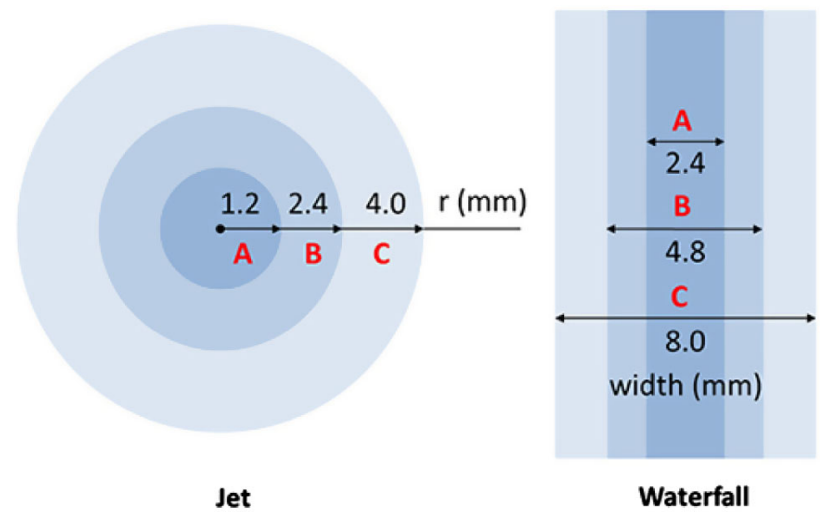

FIG. 3. Different regions for the comparison between the liquid mercury/powdery tungsten jet and the grain waterfall. The regions are marked in transverse sections of the targets $(A \in B \in C)$.

To explore the temperature rises of the target materials, the mass flow and power deposit should be figured out. Three different regions as shown in Fig. 3 are explored. In these regions, the mass flow rates are listed in Table II. The flow rates for the waterfall are the values at the beam crossing height. Actually, it makes sense to ignore the variance of the flow rate at different heights when the waterfall keeps in a compact shape. Although the velocity of the waterfall in beam-target interaction region is much smaller (around $2.6 \mathrm{~m} / \mathrm{s}$, as shown in Fig. 2) than the $20 \mathrm{~m} / \mathrm{s}$ jet velocity, it can be seen from Table II that the flow rate of the waterfall is still much higher, especially in the inner region. For a waterfall jet, apparently, the flow rate will be lower when a smaller hopper width is used. However, the power dissipation per unit of mass in the inner layer within the beam profile can be considered to be independent of the hopper width. Therefore, choosing a $3 \mathrm{~cm}$ waterfall width for the illustration and comparison of the temperature rise is fair enough.

The heat deposited (per proton) in the three regions of different targets are listed in Table III. With heat deposit data and beam current, power deposition can be calculated. Based on the detailed power dissipation information and the material flow rates in different regions, the temperature rises can be evaluated, as shown in Fig. 4. Of particular note is that, for the waterfall, the most heavily heated position

TABLE II. Mass flow rate in three different regions of the jets and the grain waterfall.

\begin{tabular}{|c|c|c|c|}
\hline \multirow[b]{3}{*}{ Region } & \multicolumn{3}{|c|}{ Mass flow rate $(\mathrm{kg} / \mathrm{s})$} \\
\hline & \multicolumn{2}{|c|}{$\mathrm{Hg} / \mathrm{W}$ jet } & \multirow{2}{*}{$\frac{\text { Grains waterfall }}{\text { Granular W }}$} \\
\hline & Liquid $\mathrm{Hg}$ & Powdery W & \\
\hline $\bar{A}$ & 1.23 & 0.87 & 21.3 \\
\hline B & 4.91 & 3.49 & 41.0 \\
\hline $\mathrm{C}$ & 13.7 & 9.70 & 62.2 \\
\hline
\end{tabular}


TABLE III. Heat deposited (per proton) in three different regions (inner beam profiles) of the jets and the grain waterfall.

\begin{tabular}{|c|c|c|c|}
\hline \multirow[b]{3}{*}{ Region } & \multicolumn{3}{|c|}{ Total energy deposit (MeV/proton) } \\
\hline & \multicolumn{2}{|c|}{$\mathrm{Hg} / \mathrm{W}$ jet } & \multirow{2}{*}{$\frac{\text { Grains waterfall }}{\text { Granular W }}$} \\
\hline & Liquid $\mathrm{Hg}$ & Powdery W & \\
\hline $\mathrm{A}$ & 283.5 & 242.3 & 403.0 \\
\hline $\mathrm{B}$ & 571.8 & 503.7 & 682.1 \\
\hline $\mathrm{C}$ & 787.9 & 708.4 & 890.8 \\
\hline
\end{tabular}

instead of the whole length along the beam direction is chosen for the comparison.

It can be seen from Fig. 4 that the temperature rises are much smaller for the grain waterfall, especially in the inner layer. As shown in Fig. 4, the estimated temperature rise for the inner layer of the liquid mercury jet is as high as more than $800^{\circ} \mathrm{C}$. For the liquid mercury jet, however, the averaged value within the whole target (the $\mathrm{C}$ region) would make more sense. With a $20 \mathrm{~m} / \mathrm{s}$ velocity, the jet just needs 0.01 second to pass the beam and the heat transfer by radiation can be ignored. Thus, the temperature rise for the mercury jet would be $\sim 210^{\circ} \mathrm{C}$. For the granular tungsten waterfall, adiabatic temperature rise for the inner layer is $\sim 180^{\circ} \mathrm{C}$. It is a small value, especially considering the high melting point of tungsten. In a word, the granular tungsten waterfall target can handle the power deposit of a $4 \mathrm{MW}$ proton beam easily.

As for the $15 \mathrm{MW}$ beam power required by the MOMENT facility, it can be evaluated that the temperature rise for the waterfall (in the inner layer) will be $\sim 675^{\circ} \mathrm{C}$, which is close to that of the dense granular flow spallation target [26] for the C-ADS project. For a granular target, this kind of temperature rise would be acceptable, which has been indicated by the prototype test using an electron beam with a power density of $\sim 130 \mathrm{~W} / \mathrm{cm}^{2}$ [39]. For the

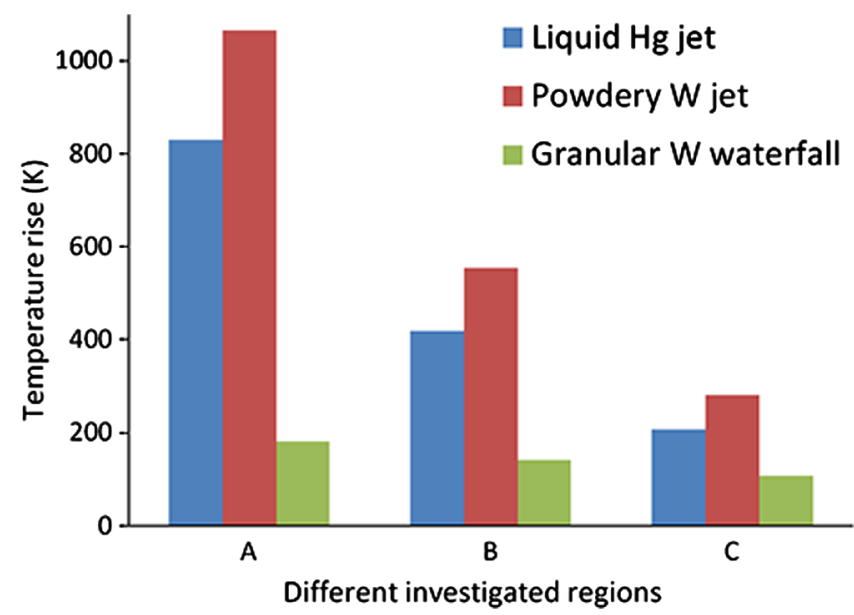

FIG. 4. Temperature rises in different regions (within beam profiles) for the three targets. construction of granular target for the Chinese initial ADS (CIADS) experimental setup which has been approved by the central government to be the second phase (1.8 B, 2016-2022) of the C-ADS project, an experimental bench is under construction to test in hydrodynamic, operation, cooling component, dust handling, granular lifting, and operation stability for the granular target system. In a word, the progress of the C-ADS target system provides valuable experiences for the R\&D of the grain waterfall target for a muon collider or a muon-decay neutrino beam facility. Considering the fact that the total power deposit in the waterfall target is around $10 \%$ of the beam power, which is much lower than that $(>50 \%)$ for the C-ADS granular flow spallation target, there will be fewer challenges for the heat exchanger of a grain waterfall target.

\section{PION AND MUON YIELDS}

For a muon collider or a muon-decay neutrino beam facility, one of the most important figures of merit concerning the performance of the target station is the numbers of the charged muons collected at the end of the pions decay channel. Using the G4BeamLine program [40], which is a particle tracking and simulation program based on the Geant 4 toolkit and specifically designed for the easy simulation of beamlines and related systems, the simulation of the production and collection of pion/muon for the granular tungsten waterfall target are performed.

Since the desired charged muons mainly come from the decay of the charged pions which are produced in the target and captured in the high-field solenoids, the calculation of the pion yields in the proton-target interaction process is substantial. In the Geant4 toolkit, different intranuclear cascade models such as BIC [41], BERTINI [42] and INCL [43] are available for the simulation of inelastic nucleonnucleus collision below $10 \mathrm{GeV}$ while the quark-gluon string (QGS) model [44] and the FRITIOF (FTF) model $[45,46]$ are used at higher energy.

In Fig. 5, the total production of charged pions (per proton per $\mathrm{GeV}$ ) produced in target body for proton beams with various kinetic energies predicted by different hadronic models are shown. The target is a tungsten bar modeled as a $40 \mathrm{~cm}$ long and $1 \mathrm{~cm}$ thick cylinder. The yields are calculated by finding the particles with a kinetic energy from $10 \mathrm{MeV}$ to $1 \mathrm{GeV}$ since too low- or too highenergy particles have little contribution to the muon flux for the neutrino beam facilities. Other than charged pions, muons of both signs are counted too.

As shown in Fig. 5, compared to the BIC and BERT model, the INCL model seems to perform a more moderate calculation. In fact, vast efforts have been spent on the highenergy extension and the improvement of the simulation of the pion production mechanism for the INCL model in recent years $[47,48]$, and reasonable agreement with the experimental data on the pion yield for proton-induced 


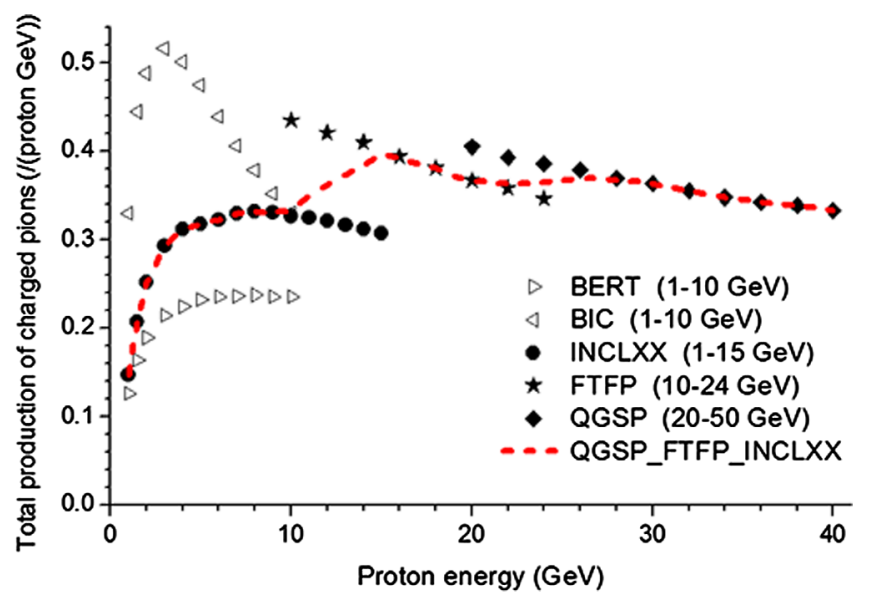

FIG. 5. Total production of charged pions (per proton per $\mathrm{GeV}$ ) produced in target body of proton beams with various kinetic energies simulated using different hadronic models.

reactions up to more than ten $\mathrm{GeV}$ has been achieved. Thus, in this paper, the INCL model is used for low incident energies and the transition to the FTF model is initiated at 9.5 GeV while the transition from the FTF model to the QGS model begins at $20 \mathrm{GeV}$. The simulation result of this model combination is shown by the dashed line.

For the simulation of the muon production, the medium density (relative factor) distributions of the granular tungsten waterfalls with 2,3 , and $4 \mathrm{~cm}$ target width are acquired from the DEM simulations, as shown in Fig. 6. The target width is defined to be the outlet width of the cubic hopper and target length has the outlet length. It can be seen that the waterfalls keep in a quite compact shape, especially in the inner regions. The beam-target intersection point is set to be at $x=0$, as shown in Fig. 2. The y-direction
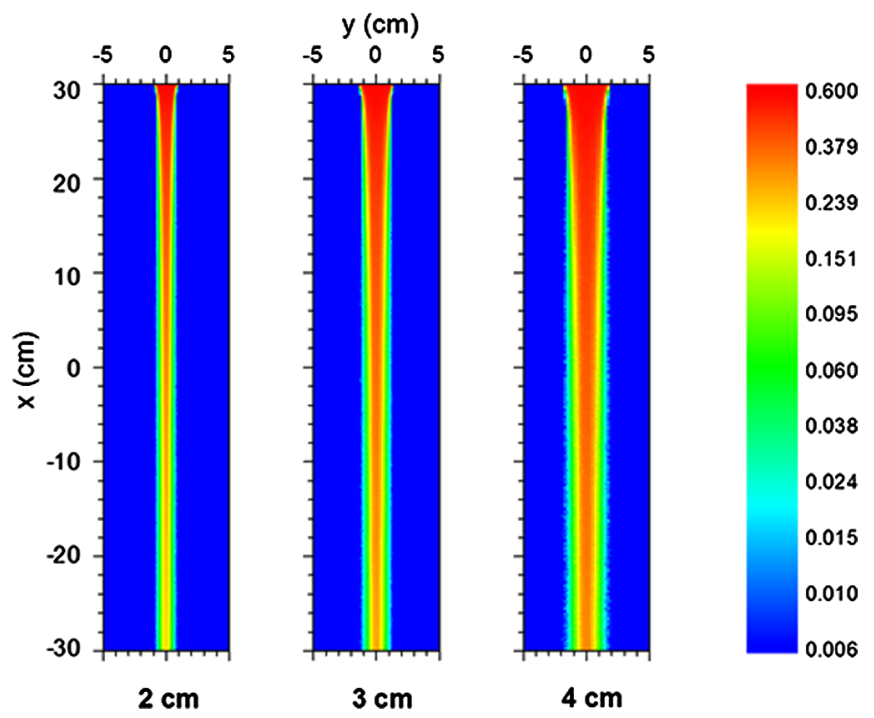

FIG. 6. Medium density (relative factor) distributions (in $x-y$ plane) for the grain waterfalls with 2,3 , and $4 \mathrm{~cm}$ target width.

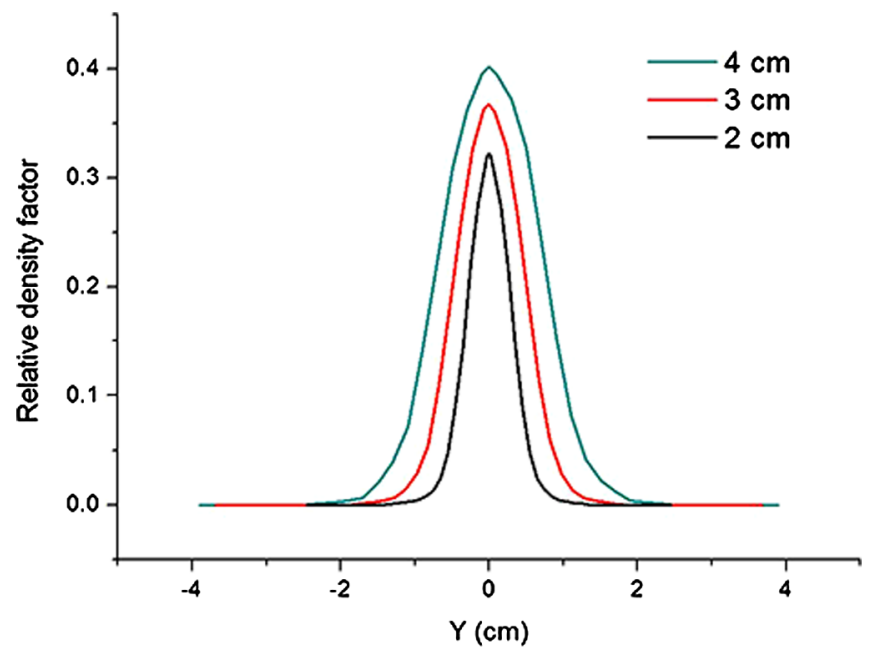

FIG. 7. Medium density (relative factor) distributions (in $\mathrm{y}$ direction, at $x=0$ height) for the grain waterfalls with 2,3 , and $4 \mathrm{~cm}$ target width.

distributions of the medium density (relative factor) at $x=0$ are shown in Fig. 7 .

Since the requirements on the muon beam for different facilities varies, here we present the calculation of the muon yields for the NF, which is a long-baseline facility, and MOMENT, which is a medium-baseline one. Using G4BeamLine, the number of the charged muons with appropriate energy and collected at the end of a $50 \mathrm{~m}$ long decay channel with a $60-\mathrm{cm}$ aperture are calculated. The magnet field adopted here is $\sim 15 \mathrm{~T}$ in the beam-target interaction region and tapers to $1.5 \mathrm{~T}$ for the $\mathrm{NF}$ and $4 \mathrm{~T}$ for MOMENT when $z>15 \mathrm{~m}$, as shown in Fig. 8 .

To focus the discussions on influences of the dimension parameters on the muon production, a tiny beam profile with a Gaussian rms radius which is 0.05 times the target width is used for the simulations in this section. Compared to the 0.15 ratio (of beam profile rms radius to jet diameter)

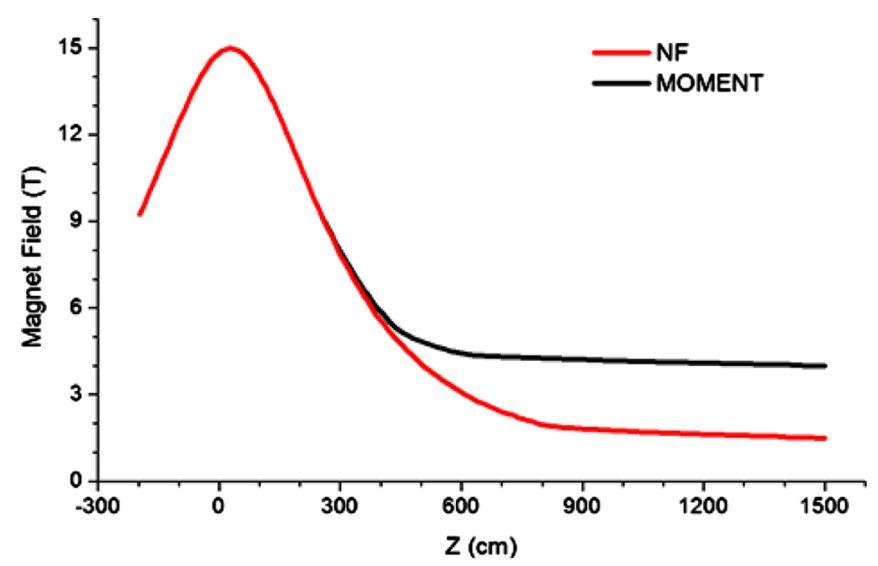

FIG. 8. Magnetic field profile distribution on axis along the field tapering section for the simulations for the NF and MOMENT. 
for the mercury jet target $[9,49]$, the value for the granular waterfall target is much smaller. The reason for adopting a much smaller ratio is that most of the grains stay in the inner layer of the waterfall, as shown in Fig. 7. When a large beam profile size (in $\mathrm{x}$ direction) is used, a large percent of the proton beam will be wasted. For small beam profile sizes, however, the muons yields are very close. Although the optimal beam profile sizes for various beamtarget parameters will be different, the following qualitative analyses of the muons yields for various waterfall dimensions will be reasonable when the same ratio (of beam profile rms radius to target width) is used.

\section{A. For the long-baseline NF}

For the neutrino factory, the charged muons and pions with kinetic energies in the range of 40 to $180 \mathrm{MeV}$ are desired, because the particles in this energy band can be efficiently captured by the downstream rf channel [50]. In the simulation studies below, the yields of the desired muons and pions at the end of the pion decay channel for the waterfall targets with different widths and lengths are calculated to figure out a proper dimension of the granular waterfall as target of the NF.

Figure 9 shows the desired (pions and) muon yields for the waterfalls with a 2, 3, and $4 \mathrm{~cm}$ width as a function of beam energy. The target lengths are $50 \mathrm{~cm}$. It can been seen that the yield rises along with beam energy from $1 \mathrm{GeV}$ to $\sim 15 \mathrm{GeV}$ with a abrupt increase after $\sim 10 \mathrm{GeV}$ and decreases after the maximum at $\sim 15 \mathrm{GeV}$ for all three widths. The abrupt increase mainly comes from the transition between the INCL and the FTF model. The relatively flat dependence of the yield on the beam energy between 4 and $9 \mathrm{GeV}$ is quite consistent with the results of the study toward the optimal beam energy for a neutrino

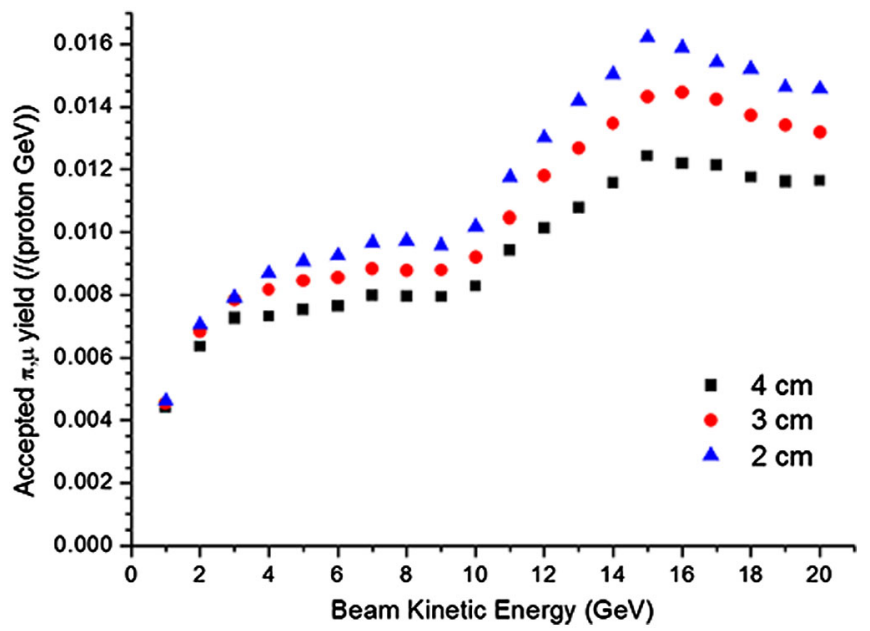

FIG. 9. Desired (pions and) muons yields (per proton per $\mathrm{GeV}$ ) for different target widths for the neutrino factory as a function of beam energy. factory and muon collider [51], which is based on experimental data from the HARP experiment [51,52].

It is also can been seen in Fig. 9 that the thinner the waterfall is, the more the desired particles can be collected for all beam energies. For example, the value at $8 \mathrm{GeV}$ for $2 \mathrm{~cm}$ width is about 0.0097 , which is $\sim 9 \%$ more than that for $3 \mathrm{~cm}$ width and $\sim 18 \%$ more than that for $4 \mathrm{~cm}$ width, as shown in Fig. 9. In spite of potential gain in (pions and) muons yields, adopting a thin waterfall gives rise to instability in beam-interaction region. For the $2 \mathrm{~cm}$ wide waterfall, in the $x=0$ region, the equivalent width is $\sim 2.3 \mathrm{~mm}$ and the width of the inner region with a relative density factor above 0.1 is no more than $10 \mathrm{~mm}$, as shown in Fig. 7, which are so small that the further reduction in waterfall width would lead to a dramatic low utilization rate of the proton beam, especially when a large transverse beam profile is used. Thus, the discussion about the muon yields for thinner waterfalls should be performed taking into consideration the beam profile.

Figure 10 shows the yields of the desired (pions and) muons of $8-\mathrm{GeV}$ beam energy for different target lengths. It can been seen that the yields are increasing up to a maximum at 30 or $40 \mathrm{~cm}$ and they are decreasing after that (according to the resolution of this analysis). The reason for that is a short target leads to a low utilization rate of proton beam, while a long one will reabsorb more pions which are captured by the magnet field. The maximum for the $2 \mathrm{~cm}$ width is less than 0.0098 at $40 \mathrm{~cm}$, which is very close to that at $50 \mathrm{~cm}$. For all target lengths except $20 \mathrm{~cm}$, it can be seen that the yields for $2 \mathrm{~cm}$ width are larger than that for 3 and $4 \mathrm{~cm}$ width. In this paper, we choose the $2 \mathrm{~cm}$ target width and $50 \mathrm{~cm}$ target length for the discussion presented in the next section, which is about the beamtarget interaction parameters for the grain waterfall as the target of the NF.

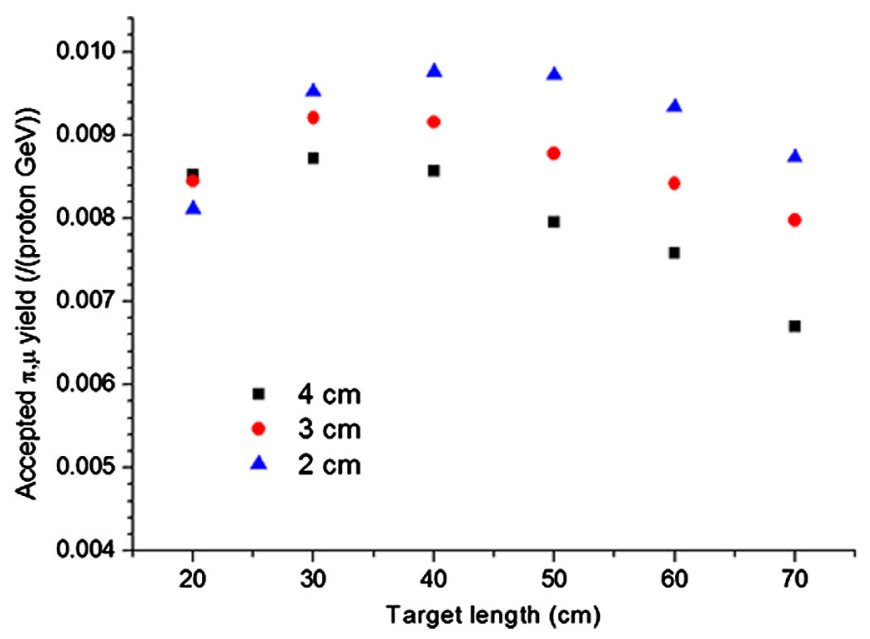

FIG. 10. Desired (pions and) muons yields (per proton per $\mathrm{GeV}$ ) of $8 \mathrm{GeV}$ proton beam for different target widths for the neutrino factory as a function of target length. 
As discussed above, after preliminary optimization of the dimensions of the granular waterfall, a muon yield of $\sim 0.01$ can be obtained at $8 \mathrm{GeV}$, which is somewhat less than that for the mercury jet target which has been carefully optimized for the NF [9]. The reduction seems reasonable due to the fact that the grain waterfall will reabsorb a larger amount of low-energy pions than liquid mercury jet. Although an enhancement in muon production can be acquired, more or less, after further optimization, the performance of the granular waterfall target may not be as good as that of a mercury jet target, especially when lowenergy muons are desired. What is worth noting is that, to a certain extent, the small equivalent width of waterfall mentioned above can make up for the penalty of the reabsorption of pions. A detailed comparison between the two target concepts which are both well optimized should be performed using the same simulation model.

\section{B. For the medium-baseline MOMENT}

As a dedicated medium-baseline facility for the $C P$ phase measurement, MOMENT is designed to produce neutrinos with energies in the range of $200-300 \mathrm{MeV}$, which come from the decay of the muons without cooling and acceleration. The muons with a momentum from 150 to $450 \mathrm{MeV} / \mathrm{c}$ are selected at the end of the pion decay channel [4].

The yields of the desired muons collected at the end of the pion decay channel for 2, 3, and $4 \mathrm{~cm}$ target widths are shown in Fig. 11. It can be seen that the yields at energies below $10 \mathrm{GeV}$ for three different target widths are very close. However, a dramatic reduction at energies around $15 \mathrm{GeV}$ for the $4 \mathrm{~cm}$ width is shown. The reason for this is that, compared to INCL, the FTF simulation above $10 \mathrm{GeV}$ gives a lower average pion momentum, as shown in Fig. 12, which results in a significantly higher reabsorption rate of the pions in the capture region for a thicker target.

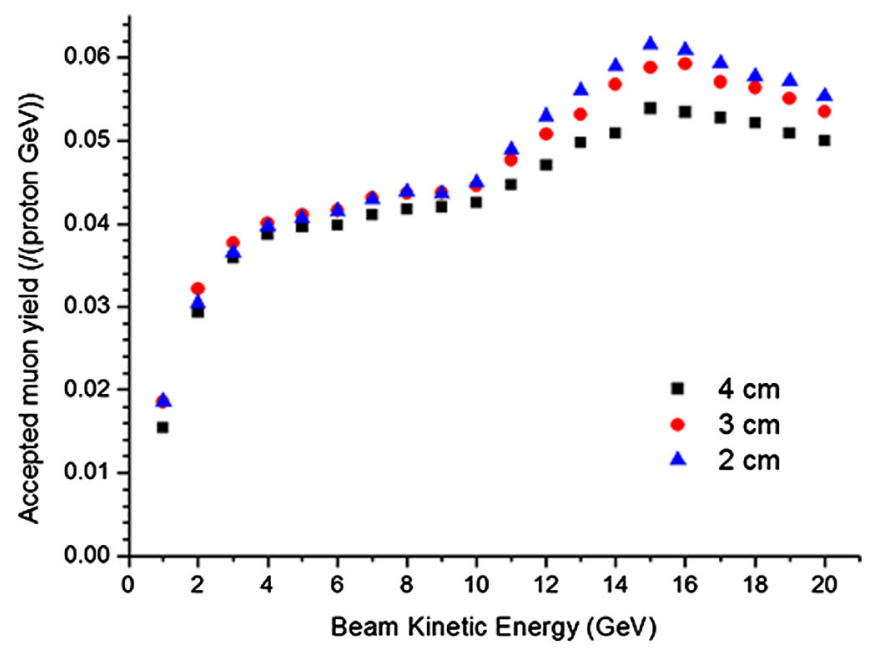

FIG. 11. Desired muons yields (per proton per $\mathrm{GeV}$ ) for different jet widths for MOMENT as a function of beam energy.

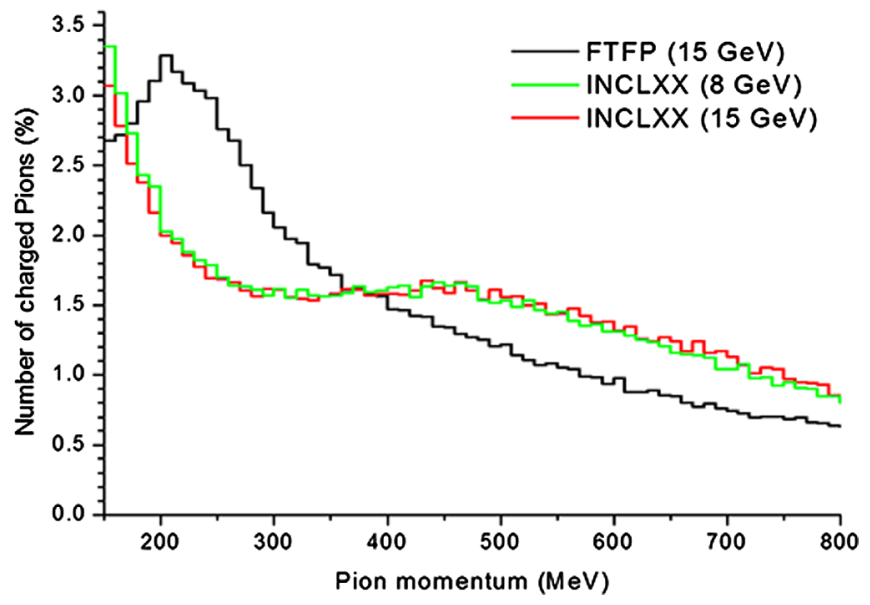

FIG. 12. The momentum distribution of the useful pions produced in the target calculated using INCL and FTF model.

As shown in Fig. 13, when only the INCL model is used, the reduction in the yields for the $4 \mathrm{~cm}$ target width stays at a relatively small value less than $6 \%$ for energies above $3 \mathrm{GeV}$, which is different from the FTF simulation. Therefore, an accurate simulation of the pions momenta is important for the optimization of the target width.

Figure 14 shows the desired muons yields of $8-\mathrm{GeV}$ proton for different target lengths. It can be seen that the most optimal lengths for 2 and $3 \mathrm{~cm}$ target width is $50 \mathrm{~cm}$ while that for $4 \mathrm{~cm}$ target width is $40 \mathrm{~cm}$. For $3 \mathrm{~cm}$ target width, the values at 40 and $50 \mathrm{~cm}$ are very close with a tiny reduction compared to that for the $2 \mathrm{~cm}$ wide and $50 \mathrm{~cm}$ long waterfall. When the muon yields are close, the larger target is always a better choice for a higher flow rate. Therefore, $3 \mathrm{~cm}$ width and $50 \mathrm{~cm}$ length are chosen for the discussion in the next section.

As mentioned above, the MOMENT will adopt a cw superconducting linac. The beam power will be $15 \mathrm{MW}$ and

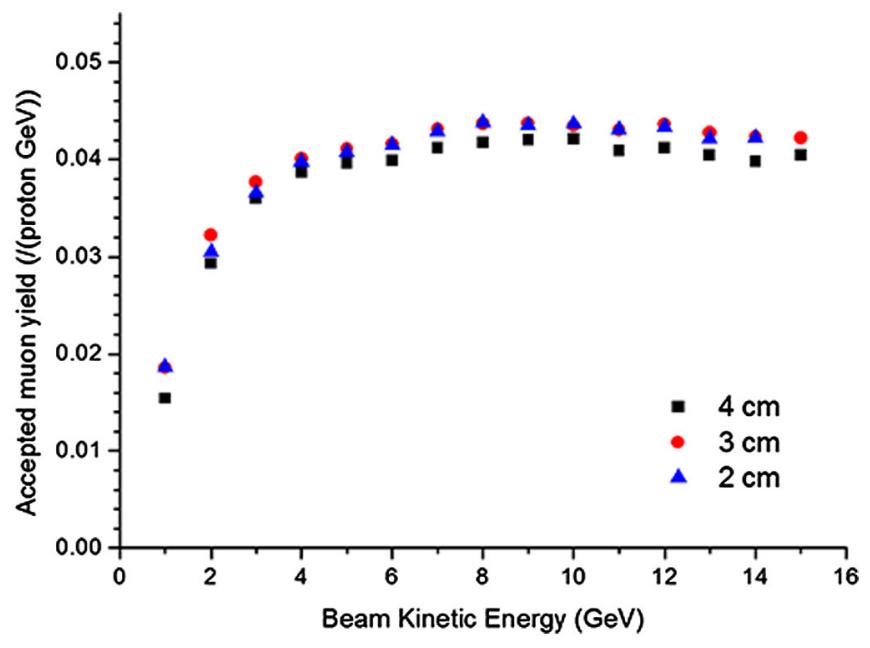

FIG. 13. Same as for Fig. 11 except for choosing INCL model for all simulation. 


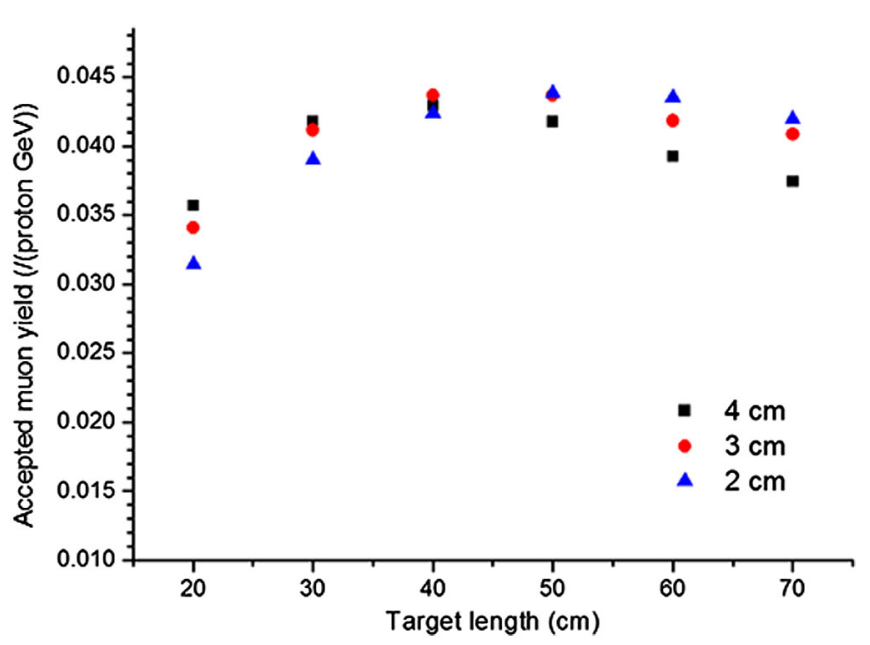

FIG. 14. Desired muons yields (per proton per $\mathrm{GeV}$ ) of $8 \mathrm{GeV}$ proton beam for different outlet widths for MOMENT as a function of target length.

the beam energy is still under optimization depending on the muon production efficiency and the cost [4]. In the nominal design, $1.5 \mathrm{GeV}$ in energy and $10 \mathrm{~mA}$ in current are used [13]. For the waterfall target, as can be seen from the simulation results presented above, a beam energy around or above $8 \mathrm{GeV}$ seems to be a good choice for a high production efficiency of charged muons, ignoring the high values at high energies above $10 \mathrm{GeV}$ predicted by different models. Therefore these preliminary studies show that is reasonable to use an $8-\mathrm{GeV}$ beam energy instead of $1.5 \mathrm{GeV}$ one for MOMENT. In that case a longer linac is needed while the technical challenges posed by a high current will be reduced. Careful consideration is required to decide the detailed configuration of the linac.

\section{BEAM-JET INTERACTION PARAMETERS}

Due to the limitation on the target dimension for a low reabsorption rate of the charged pions, there will be quite a significant proportion of primary beam protons which need to be guided to a beam dump after the beam-target interaction. Usually, a grain collection pool is well designed to serve as the proton beam dump at the same time $[3,4,9]$ since the feasibility of guiding the spent proton to pass through the solenoids gaps to an outer beam dump is still under study [4]. For a granular waterfall target, the grain collection pool should be located at a position downstream from the beam-target interaction region as close as possible to facilitate the flowing of grains in the inclined chute between the waterfall and the pool. Therefore, the beamtarget interaction parameters including the launch direction and position of the proton beam should be carefully optimized giving consideration to both muon yield and beam dumping.

The beam-target interaction geometry for the waterfall target is shown in Fig. 15. The beam launch position is

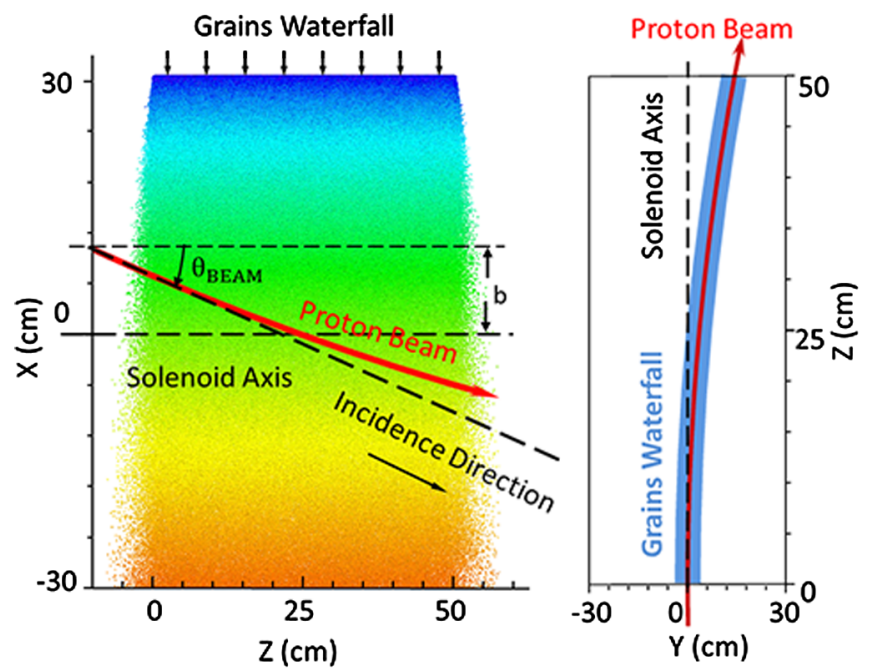

FIG. 15. The beam-target geometry for the waterfall target.

defined to be the intersection point of the beam and the $z=-10 \mathrm{~cm}$ plane. The launch parameter $\mathrm{b}$ is defined as the distance between the launch position and the solenoid axis while $\theta_{\text {BEAM }}$ is the launch angle of the proton beam. To figure out the influence of the beam-target interaction parameters on the muon yield, a series of simulations are performed. The kinetic energy of the proton beam is $8 \mathrm{GeV}$. The beam profile is uniform within $1 \mathrm{~cm}$ in $\mathrm{x}$ direction and Gaussian in $\mathrm{y}$ direction with a rms y value 0.05 times the target width. The $2 \mathrm{~cm}$ wide and $50 \mathrm{~cm}$ long target is used for the NF while the $3 \mathrm{~cm}$ wide and $50 \mathrm{~cm}$ long one is chosen for MOMENT, as mentioned above.

Of particular note is that a cambered waterfall as shown in Fig. 15 is used here. According to the curved track of the proton beam launched with an angle relative to the magnetic field, the curved hopper outlet were properly shaped to form a cambered waterfall. Obviously, the utilization rate of the tilted proton beam for such a kind of waterfall will be higher than that for a plane one, especially when the launch angle is large.

As shown in Table IV and V, the muon yields for the NF and MOMENT with different beam-target interaction parameters were calculated. It can be seen that the increase of the launch angle generally results in a reduction in muon yield. For different launch angles, the optimal value of the

TABLE IV. Muon yields for the NF with different beam-target interaction parameters.

\begin{tabular}{lccccc}
\hline \hline & \multicolumn{5}{c}{ Muon yields (pppGeV) $\times 1000$ (for different b) } \\
\cline { 2 - 6 }$\theta_{\text {Beam }}(\mathrm{mrad})$ & $0.0 \mathrm{~cm}$ & $3.5 \mathrm{~cm}$ & $7.0 \mathrm{~cm}$ & $10.5 \mathrm{~cm}$ & $14.0 \mathrm{~cm}$ \\
\hline 0 & 9.586 & 8.968 & - & - & - \\
100 & 9.088 & 9.500 & 8.743 & - & - \\
200 & 8.543 & 9.104 & 9.296 & 8.629 & - \\
300 & 7.735 & 8.110 & 8.828 & 8.944 & 8.379 \\
\hline \hline
\end{tabular}


TABLE V. Muon yields for MOMENT with different beamtarget interaction parameters.

\begin{tabular}{lccccc}
\hline \hline & \multicolumn{5}{c}{ Muon yields (pppGeV) $\times 100$ (for different b) } \\
\cline { 2 - 6 }$\theta_{\text {Beam }}(\mathrm{mrad})$ & $0.0 \mathrm{~cm}$ & $3.5 \mathrm{~cm}$ & $7.0 \mathrm{~cm}$ & $10.5 \mathrm{~cm}$ & $14.0 \mathrm{~cm}$ \\
\hline 0 & 4.326 & 4.251 & - & - & - \\
100 & 4.211 & 4.240 & 4.170 & - & - \\
200 & 4.043 & 4.197 & 4.151 & 4.081 & - \\
300 & 3.753 & 3.884 & 4.021 & 3.991 & 3.887 \\
\hline \hline
\end{tabular}

launch parameter varies. In general, the launch position that ensures an approximate axial symmetry of the beam track would be the best choice.

To figure out the requirement on the pool location for a feasible design of the grain collection channel, the study on the flow in the chute after the waterfall is still ongoing. In fact, preliminary studies of the inclined granular flows fed by a reservoir have been performed [53]. According to our preliminary studies, an inclined chute angle larger than $15^{\circ}$ will be feasible for the granular flow to move smoothly [53]. As shown in Tables IV and V, owing to the adoption of the cambered waterfall, the reduction in muons yield can be limited to less than $7 \%$, even using an angle of $300 \mathrm{mrad}$ $\left(\sim 17.2^{\circ}\right)$. Thus, the conceptual design of the grain stream collection channel and the beam dumping arrangement as shown in Fig. 1 is reasonable. In addition, a careful design of the collection channel for the grains would bring about additional pions by making the granular flow in the chute act as a second target.

\section{DISCUSSION AND CONCLUSIONS}

The studies of the working mechanism and the power handling ability of the grain waterfall target based on the conceptual design presented in this paper indicate that this kind of target solution has the potential to meet the requirements of the MOMENT which is driven by a cw beam power as high as $15 \mathrm{MW}$. In addition, compared to the liquid metal jet, the grain waterfall also possesses more advantages to act as the target of a muon collider or a muondecay neutrino beam facility, owing to its simplicity and reliability.

More detailed optimizations of the parameters for the waterfall target of the MOMENT would make for some gains in muons yield. Compared to the mercury jet target, the gravity-driven granular waterfall target can be designed with more flexibility. For example, the curvature of the cambered waterfall can vary along with the beam energy; the height of the beam-target interaction region should be further optimized; a higher ratio of the hopper outlet width to the granular diameter can be adopted to acquire a more compact waterfall to mitigate the interruptions to the helical motions of the charged pions.

Due to the fact that the grain waterfall will cross through the magnetic field, when pure tungsten is used for the grains, the influences from the electromagnetic induction and quadrupole effects would be more significant than that for the liquid metal jet target, in spite of a smaller waterfall velocity. However, unlike the liquid metal jet, the granular waterfall has an essential advantage in material components choice. By adopting a material component with low electric conductivity and magnetic susceptibility, the shape transformations and instabilities of the grain waterfall in strong magnetic field can be avoided. Thus, another important subject in the detailed design work for the waterfall target of the MOMENT is the choice of the material components of the grains. The fabrication and experimental studies of the granular material are underway by the target team of C-ADS and the MOMENT group. For the moment, tungsten carbide seems to have the potential to meet the requirements owing to its extremely low magnetic susceptibility and electric conductivity. Moreover, with high melting point, high density, great hardness, favorable thermal conductivity, and excellent high-temperature strength, the grains made of tungsten carbide can also work properly in the grains circuit of the target system.

In addition to the material choice, another strategy for the mitigation of the electromagnetic effects is the reduction in the magnetic field strength. Unlike a liquid jet, there will be no Richtmyer-Meshkov instability for a grain waterfall. Thus, a strong magnetic field as strong as 15 even $20 \mathrm{~T}$ is not necessary. With a bore radius of $100 \mathrm{~cm}$ for the capture/ decay channel aperture, a magnetic field of less than 1 or $2 \mathrm{~T}$ would work for the NF or MOMENT, respectively. With these low magnetic fields, the influences to the stream of the grain should be much slighter.

\section{ACKNOWLEDGMENTS}

The discussions within the target team of the C-ADS project and the MOMENT group are encouraging the finishing of the work. Valuable support from the IMP linac center are also gratefully acknowledged. This work was supported by the National Magnetic Confinement Fusion Science Program of China (Grant No. 2014 GB104002), the Strategic Priority Research Program of the Chinese Academy of Sciences (Grant No. XDA03030100), and the National Natural Science Foundation of China (Grant No. 11527811).

[1] S. Geer and M. S. Zisman, R\&D PROPOSAL FOR THE NATIONAL MUON ACCELERATOR PROGRAM, Report No. FERMILAB-TM-2459-APC, 2010, http:// escholarship.org/uc/item/1j074034.

[2] S. Geer, Neutrino beams from muon storage rings: Characteristics and physics potential, Phys. Rev. D 57, 6989 (1998).

[3] M. Bogomilov et al., Neutrino factory, Phys. Rev. ST Accel. Beams 17, 121002 (2014). 
[4] J. Cao, M. He, Z.-L. Hou, H.-T. Jing, Y.-F. Li, Z.-H. Li, Y.-P. Song, J.-Y. Tang, Y.-F. Wang, Q.-F. Wu, Y. Yuan, and Y.-H. Zheng, Muon-decay medium-baseline neutrino beam facility, Phys. Rev. ST Accel. Beams 17, 090101 (2014).

[5] H. G. Kirk et al., Targetry for a $\mu^{+} \mu^{-}$collider, in Proceedings of the 18rd Particle Accelerator Conference, New York, USA, 1999 (IEEE, Piscataway, NJ, 1999), p. 3029.

[6] A. Hassenein et al., The primary target facility for a neutrino factory based on muon beams, in Proceedings of the 19rd Particle Accelerator Conference, Chicago, USA, 2001 (IEEE, Piscataway, NJ, 2001), p. 1583.

[7] K. T. McDonald et al., THE MERIT HIGH-POWER TARGET EXPERIMENT AT THE CERN PS, in Proceedings of the 23rd Particle Accelerator Conference, Vancouver, Canada, 2009 (IEEE, Piscataway, NJ, 2009), p. 795.

[8] X. Ding et al., A PION PRODUCTION AND CAPTURE SYSTEM FOR A $4 \mathrm{MW}$ TARGET, in Proceedings of the Ird International Particle Accelerator Conference, Kyoto, Japan, 2010 (ICR, Kyoto, Japan, 2010), p. 4272.

[9] J. J. Back, C. Densham, R. Edgecock, and G. Prior, Particle production and energy deposition studies for the neutrino factory target station, Phys. Rev. ST Accel. Beams 16, 021001 (2013).

[10] V. Graves et al., Systems testing of a free hg jet system for use in a high-power target experiment, in Proceedings of the 22rd Particle Accelerator Conference, Albuquerque, USA, 2007 (IEEE, Piscataway, NJ, 2007), p. 3136.

[11] H. G. Kirk et al., The MERIT High-Power Target Experiment at the CERN PS, in Proceedings of the 11rd European Particle Accelerator Conference, Genoa, Italy, 2008 (EPS-AG, Italy, 2008), p. 2886.

[12] S. Choubey et al., Interim Design Report for the International Design Study for a Neutrino Factory, Report No. FERMILAB-PUB-11-581-APC, 2011, http://www .osti.gov/scitech/biblio/1029650.

[13] Z.-H. Li and J.-Y. Tang, A CW superconducting linac as the proton driver for a medium baseline neutrino beam in China, Chin. Phys. C 38, 127001 (2014).

[14] R. Samulyak and Y. Prykarpatskyy, Richtmyer-Meshkov instability in liquid metal flows: influence of cavitation and magnetic fields, Math. Comput. Simul. 65, 431 (2004).

[15] H. G. Kirk et al., A PROOF-OF-PRINCIPLE EXPERIMENT FOR A HIGH-POWER TARGET SYSTEM, in Proceedings of the 10rd European Particle Accelerator Conference, Edinburgh, Scotland, 2006 (EPS-AG, Scotland, 2006), p. 3254.

[16] V. Hassanein et al., Thermoelastic response of suddenly heated liquid targets in high-power colliders, in Proceedings of the 19rd Particle Accelerator Conference, Chicago, USA, 2001 (IEEE, Piscataway, NJ, 2007), p. 624.

[17] A. Fabich, High power proton beam shocks and magnetohydrodynamics in a mercury jet target for a neutrino factory, Doctoral dissertation, Tech. U. Wienna, 2002.

[18] H. Park, T. Tsang, H. G. Kirk, F. Ladeinde, V. B. Graves, P. T. Spampinato, A. J. Carroll, P. H. Titus, and K. T. McDonald, Optical diagnostics of mercury jet for an intense proton target, Rev. Sci. Instrum. 79, 045111 (2008).

[19] Z. Li, P. Cheng, H. Geng, Z. Guo, Y. He, C. Meng, H. Ouyang, S. Pei, B. Sun, J. Sun et al., Physics design of an accelerator for an accelerator-driven subcritical system, Phys. Rev. ST Accel. Beams 16, 080101 (2013).

[20] A. Fabich and J. Lettry, Experimental observation of proton-induced shocks and magneto-fluid-dynamics in liquid metal, Nucl. Instrum. Methods Phys. Res., Sect. A 503, 336 (2003).

[21] S. Zhang, P. Lin, C.-L. Wang, Y. Tian, J.-F. Wan, and L. Yang, Investigating the influence of wall frictions on hopper flows, Granular Matter 16, 857 (2014).

[22] P. Lin, S. Zhang, J. Qi, Y. Xing, and L. Yang, Numerical study of free-fall arches in hopper flows, Physica A (Amsterdam) 417, 29 (2015).

[23] Y. Tian, P. Lin, S. Zhang, C. Wang, J. Wan, and L. Yang, Study on free fall surfaces in three-dimensional hopper flows, Advanced powder technology : the international Journal of the Society of Powder Technology, Japan 26, 1191 (2015).

[24] J.-F. Wan, S. Zhang, Y. Tian, P. Lin, G. Yang, and L. Yang, Influence of geometrical and material parameters on flow rate in simplified ADS dense granular-flow target: a preliminary study, J. Nucl. Sci. Technol. 53, 1809 (2016).

[25] X. Zhang, S. Zhang, G. Yang, P. Lin, Y. Tian, J.-F. Wan, and L. Yang, Investigation of flow rate in a quasi-2D hopper with two symmetric outlets, Phys. Lett. A 380, 1301 (2016).

[26] L. Yang and W.-L. Zhan, New concept for ADS spallation target: Gravity-driven dense granular flow target, Sci. China: Technol. Sci. 58, 1705 (2015).

[27] Y. Tian, J. Lai, L. Yang, J. Qi, and Q. Zhou, A heterogeneous CPU-GPU implementation for discrete elements simulation with multiple GPUs, in Proceedings of the 2013 International Joint Conference on Awareness Science and Technology and Ubi-Media Computing, Aizuwakamatsu, Japan 2013 (IEEE, Aizuwakamatsu, 2014), p. 547.

[28] J. Qi, K.-C. Li, H. Jiang, Q. Zhou, and L. Yang, GPU-accelerated DEM implementation with CUDA, Int. J. Comput. Sci. Eng. 11, 330 (2015).

[29] P. A. Cundall and O. D. L. Strack, A discrete numerical model for granular assemblies, Geotechnique 29, 47 (1979).

[30] P. A. Cundall and O. D. L. Strack, A discrete numerical model for granular assemblies - reply, Geotechnique 30, 335 (1980).

[31] H. Tao et al., Discrete element method modeling of nonspherical granular flow in rectangular hopper, Chemical Engineering and Processing: Process Intensification 49, 151 (2010).

[32] Y. Yu and H. Saxn, Discrete element method simulation of properties of a 3D conical hopper with mono-sized spheres, Advanced powder technology : the international Journal of the Society of Powder Technology, Japan 22, 324 (2011).

[33] H. Nakashima, Y. Shioji, T. Kobayashi, S. Aoki, H. Shimizu, J. Miyasaka, and K. Ohdoi, Determining the angle of repose of sand under low-gravity conditions using discrete element method, Journal of terramechanics 48, 17 (2011).

[34] H. Mio, M. Kadowaki, S. Matsuzaki, and K. Kunitomo, Development of particle flow simulator in charging process of blast furnace by discrete element method, Minerals engineering 33, 27 (2012). 
[35] B. C. Burman, A discrete numerical model for granular assemblies, Geotechnique 30, 331 (1980).

[36] K. L. Johnson, Contact Mechanics (Cambridge University Press, Cambridge, England, 1987).

[37] M. P. Allen and D. J. Tildesley, Computer Simulation of Liquids (Oxford University Press, New York, 1989).

[38] S. Agostinelli et al., Geant4-a simulation toolkit, Nucl. Instrum. Methods Phys. Res., Sect. A 506, 250 (2003).

[39] W. L. Zhan et al., ACCELERATOR DRIVEN SUSTAINABLE FISSION ENERGY, in Proceedings of the $7 \mathrm{rd}$ International Particle Accelerator Conference, Kyoto, Japan, 2016 (ICR, Busan, Korea, 2016), p. 4271.

[40] T.J. Roberts et al., G4BEAMLINE PARTICLE TRACKING IN MATTER DOMINATED BEAM LINES, in Proceedings of the 24th Particle Accelerator Conference, PAC-2011, New York, 2011 (IEEE, New York, 2011), pp. 373-375.

[41] G. Folger, V. N. Ivanchenko, and J. P. Wellisch, The Binary Cascade, Eur. Phys. J. A 21, 407 (2004).

[42] H. W. Bertini, Low-Energy Intranuclear Cascade Calculation, Phys. Rev. 131, 1801 (1963).

[43] A. Boudard, J. Cugnon, S. Leray, and C. Volant, Intranuclear cascade model for a comprehensive description of spallation reaction data, Phys. Rev. C 66, 044615 (2002).

[44] N. S. Amelin, V. D. Toneev, and K. K. Gudima, Quark Gluon String Model and Ultrarelativistic Heavy Ion Interactions, Sov. J. Nucl. Phys. 51, 327 (1990).

[45] B. Andersson, G. Gustafson, and B. Nilsson-Almqvist, A model for low-pT hadronic reactions with generalizations to hadron-nucleus and nucleus-nucleus collisions, Nucl. Phys. B281, 289 (1987).
[46] B. Nilsson-Almqvist and E. Stenlund, Interactions between hadrons and nuclei: The Lund Monte Carlo - FRITIOF version 1.6, Comput. Phys. Commun. 43, 387 (1987).

[47] S. Pedoux and J. Cugnon, Extension of the Liège intranuclear cascade model at incident energies between 2 and $12 \mathrm{GeV}$. Aspects of pion production, Nucl. Phys. A866, 16 (2011).

[48] D. Mancusi, A. Boudard, J. Cugnon, J.-C. David, P. Kaitaniemi, and S. Leray, Extension of the Liège intranuclear-cascade model to reactions induced by light nuclei, Phys. Rev. C 90, 054602 (2014).

[49] X. Ding, J. S. Berg, D. Cline, and H. G. Kirk, Optimization of a mercury jet target for a neutrino factory or a muon collider, Phys. Rev. ST Accel. Beams 14, 111002 (2011).

[50] X. Ding, D. Cline, H. Kirk, and J. Berg, OPTIMIZED PARAMETERS FOR A MERCURY JET TARGET, in Proceedings of the 23rd Particle Accelerator Conference, Vancouver, Canada, 2009 (IEEE, Piscataway, NJ, 2009), p. 2748 .

[51] J. Strait, N. Mokhov, and S. Striganov, Towards the optimal energy of the proton driver for a neutrino factory and muon collider, Phys. Rev. ST Accel. Beams 13, 111001 (2010).

[52] M. G. Catanesi, E. Radicioni, R. Edgecock, M. Ellis, F. Soler, C. Gößling, S. Bunyatov, A. Krasnoperov, B. Popov, V. Serdiouk et al., Large-angle production of charged pions with 3-12.9 GeV/c incident protons on nuclear targets, Phys. Rev. C 77, 055207 (2008).

[53] G. Yang, S. Zhang, P. Lin, Y. Tian, J.-F. Wan, and L. Yang, Numerical and experimental verification of a damping model used in DEM, Granular Matter 18, 1 (2016). 